\title{
Lagrange's multiplier based resource management for energy efficient D2D communication in 5G networks
}

\author{
Krishna Pandey $^{1} \cdot$ Rajeev Arya $^{1}$ (1) $\cdot$ Sandeep Kumar $^{2}$
}

Received: 13 September 2020/Revised: 2 December 2020/ Accepted: 10 December 2020

(C) The Society for Reliability Engineering, Quality and Operations Management (SREQOM), India and The Division of Operation and Maintenance, Lulea University of Technology, Sweden 2021

\begin{abstract}
Device to device communication is the predominantly renowned trait for the $5 \mathrm{G}$ network and IoT applications. In the work, proposed novel joint low power/energy efficient resource allocation with mode selection for the D2D communication underlay in-band with transmit power, interference, data rate constraints are investigated with formulation of a novel problem which integrates the three major modules (resource management, mode selection, and power management) of D2D communication into one. To achieve the low power/energy efficient resource allocation with mode selection, we formulate novel optimization problem with objective of maximizing the energy efficiency using the subtractive form method to solve fractional objective function and form an iterative algorithm. The formulated fractional optimization problem is transformed into min-max problem and solved by the Lagrange dual function with low transmit power, interference, data rate constraints as a lagrange multipliers via an iterative process to achieve the optimal low power. Numerical analysis exemplifies and validates the optimal low power and the energy efficient characteristics of the novel proposed algorithm with all constraints to ensure the quality of the
\end{abstract}

Rajeev Arya

rajeev.arya@nitp.ac.in

Krishna Pandey

krishnapnitp@gmail.com

Sandeep Kumar

sandeep@nitk.edu.in

1 Department of Electronics and Communication Engineering, National Institute of Technology Patna, Patna, Bihar 800005, India

2 Department of Electronics and Communication Engineering, National Institute of Technology Karnataka, Surathkal, Mangalore, Karnataka 575025, India communication for the D2D communication, 5G, and IoT applications with the industrial need of low power/energy efficient devices to promote the conservation of energy and green communication.

Keywords D2D communication - IoT - 5G - Fractional optimization · Min-max problem · Lagrange's dual function

\section{Introduction}

To provide the large connectivity and applications like digital cities, smart agriculture, and intelligent healthcare, 5G becomes the integral part of the Internet of Things (IoT). To ensure the quality of service in the IoT applications the $5 \mathrm{G}$ acts as driving technology (Chettri and Bera 2020). In 5G and next generation cellular networks, the energy efficiency is a major factor which improves the overall performance. The device to device communication (D2D) ensures the energy efficiency in the wireless networks through different energy efficient algorithm and protocols. Due to its promising feature, the D2D communication becomes the subpart of $5 \mathrm{G}$ networks technologies (Hoyhtya et al. 2018).

In the present days, the numbers of IoT application are available in health sectors. In the pandemic COVID-19 situation, Indian government launched an Application named as Arogya Setu app to nurse the social distance by gathering the data of nearby surrounded persons. With keep in view of this problem, in the Arogya Setu app (an App by Indian government for gathering the information of and give awareness to the people during fight COVID-19), when we tried to use this application, the Bluetooth-ON prerequisite condition is imperative. But for these types of application, 
reliability and accessibility is exceedingly focal concern. Bluetooth requirement also drained the battery into some extent. To solve this problem, we have to provide a communication platform which ought to perform in licensed domain network and retain the power consumption. In our point of view device 2 device communication is probable solution for the above said problem.

We are highly motivated from this problem and try to give the solution through start working in the domain of D2D communication. As we studied that the energy efficient, low power device is always a concern for the purpose of communication, we work on the field of D2D communication and propose a novel Joint Low power and energy efficient resource management algorithm with mode selection for D2D communication in 5G and IoT application.

\section{Related work}

With the ingenious attribute and energy efficient spirit of D2D communication several underlay-in band cellular network models are optimized and analyzed to attain the resource allocation, mode selection, and power control strategies individually and jointly. For the mode selection the objective formulations are done based on maximization of quality of services (QoS) with the minimum transmit power (Shalmashi et al. 2014). A mode selection through the concept of guardzone is proposed for the D2D communication network (Ye and Zhang 2015). Load balancing technique is used in the optimal mode selection by proposing the distributed algorithm (Liu 2016). Using the bandit model for the mode selection is done for the small cell D2D communication (Maghsudi and Niyato 2017). The resource management with dynamic power control for D2D communication shown using the Lagrangian decomposition method for optimal power allocation is illustrated (Jiang et al. 2018). For the optimal power control, the reuse of the orthogonal pilots describes the concept of sharing of resources (Ghazanfari et al. 2018). A joint algorithm mode selection, channel allocation and power assignment is represented to minimize the power consumption with the data rate requirement (Gao et al. 2014). A joint admission control, mode selection and power management problem is proposed to enhance the throughput with QoS and interference constraint (Azam 2016). The maximization problem in D2D femtocell with considering the mode selection, resource allocation and power assignment is represented and solved by using the Lagrangian dual composition method (Wang et al. 2016). Maximization of the energy efficiency is a significant paradigm of the D2D communication.

After studying and analyzing the above mentioned and energy efficient articles (Fodor et al. 2016; Han et al. 2011, 2019; Feng et al. 2013; Gandotra et al. 2017; Wang et al. 2014, 2018; Sheng et al. 2016; Xu et al. 2016; Cai et al.
2019; Awan et al. 2020; Lee and Lee 2019; Huang et al. 2019), we conclude that the several authors already epitomize the concept of energy efficient algorithms for the mode selection, resource allocation and power control individually or jointly. In the above-mentioned glimpse of the literatures, Shalmashi et al. (2014), Ye and Zhang (2015), Liu (2016), Maghsudi and Niyato (2017) represent the study of different methods of mode selection, literature (Maghsudi and Niyato 2017; Jiang et al. 2018; Han et al. 2019) discuss about the resource allocation with optimized power. The Joint algorithm with mode selection and channel allocation was done with optimal power distribution in Gao et al. (2014), Azam (2016), Wang et al. (2016). The data rate (Gao et al. 2014) and interference (Azam 2016) are the widely and singly used constraint for achieving the optimal results, via using Lagrange dual decomposition method (Wang et al. 2016) and it has been observed after literature review that several authors discuss about the different power control methods to achieve the low power and energy efficiency separately to resolve the mode selection and resource management issues. There is no work for building a model which ensures the optimal low power requirement with energy efficiency by including all (data rate, interference, low transmit power) constraints together with performing the mode selection, and resource allocation for the D2D communication.

In this paper, we study the individual and joint association of resource allocation, mode selection and power allocation for the energy efficient D2D communication. We propose a novel Joint Low power and energy efficient resource management algorithm with mode selection. To create the proposed algorithm, we formulate the combinatorial fractional optimization problem for the energy efficient D2D communication to address the resource allocation, mode selection and power control issues jointly. Mode selection is done by using the basic algorithm using probabilistic approach. For the attaining the maximum energy efficient value, iterative approach is applied to fractional objective function which is represented in the subtracted form. In each iteration the optimal power value with resource allocation with mode selection is achieved to maximize the energy efficiency (EE) and further Lagrange dual function having Lagrange multipliers optimize the power with low transmit power rate, data rate, and interference constraints as a Min-Max problem.

To propose the Joint Low power energy efficient resource management algorithm with mode selection, we utilize the concept of energy efficiency and Min-Max approach (Jiang et al. 2016; Mensah et al. 2016). The footprint of our proposed algorithm after the profound literature survey is mentioned below.

1. Formulate the novel problem which integrates the three problems mode selection, resource allocation and optimal power into one. 
2. Propose the novel mode selection method with theorem for the D2D communication.

3. Use the concept of maximizing the energy efficiency (Jiang et al. 2016; Dinkelbach 1967)

4. Add the low transmit power rate, data rate, and interference constraints to the formulated problem to transform into Min-Max problem.

5. With help of Lagrange dual function and Lagrange multipliers (Mensah et al. 2016) concept (adding interference constraint), we further obtain the optimal transmit power with low transmit power rate, data rate, and interference constraints.

6. Finally, we propose novel joint Low power and energy efficient resource management algorithm with mode selection.

7. By evaluating the numerical results, we finally attain a framework which reflects the improvement in the related work of energy efficient resource management algorithms (Jiang et al. 2016) by keeping the view of Low power devices and power conservation with maximum energy efficiency.

The paper is organized as follows. Section 2 describes the formulated problem with system model. In Sect. 3, the proposed Joint Low power and energy efficient resource management algorithm with mode selection is presented. The algorithm for the mode selection, iterative algorithm for the maximum energy efficiency and the iterative algorithm using Lagrange dual function with Lagrange multipliers are described here. The numerical results using system parameters evaluate the different performance parameters of the proposed algorithm in Sect. 4. And, Sect. 5 concludes the proposed work with improvement in the past analogous efforts.

\section{System model and problem formulation}

We assume the uplink resources of cellular user equipment (CUE) are shared by the D2D user pairs (DU) and there is a random distribution of DU and CUE in the cell. In the single cell we consider that

$$
\begin{aligned}
& \mathrm{A}=\left\{1,2,3, \ldots \mathrm{A}_{\text {cue }}\right\} \\
& \mathrm{B}=\{1,2,3, \ldots, \mathrm{B}\} \\
& \mathrm{C}=\{1,2,3, \ldots, \mathrm{t}\} \\
& \mathrm{D}=\left\{1,2,3 \ldots \mathrm{t}+\mathrm{A}_{\text {cue }}\right\} \\
& \text { must satisfy that } \mathrm{t} \leq \mathrm{A}_{\text {cue }}=\mathrm{B} \text { and } \mathrm{D}=\{\mathrm{AUC}\}
\end{aligned}
$$

where $\mathrm{A}, \mathrm{B}, \mathrm{C}$ denotes the set of CUE's and the resource blocks $R_{B}$ and $D 2 D$ users DU's respectively, we assume that 1 time frequency resource block consists of one-time slot and 4 frequency subcarriers and jth resource block allocated to cellular user equipment are reused by the ithe mobile user MU with nth mode selection. Let us assume that $G_{i j}$ is the transmit power of the transmitter of the ith mobile user MU on the jth resource block $R_{B}$ and $G_{b}$ denotes the base station transmission power. Let $\mathrm{T}_{\mathrm{DU}}$ and $\mathrm{T}_{\mathrm{CUE}}$ represent the data rate of DU and CUE respectively. By using the Shannon capacity formulae (Fan et al. 2017), we evaluate the mathematical formulae of $\mathrm{T}_{\mathrm{DU}}$ and $\mathrm{T}_{\mathrm{CUE}}$ (Fig. 1)

$T_{C U E}\left(G_{i j}\right)=B \log _{2}\left(1+\frac{G_{b} h d_{b}^{-\alpha}}{\sum_{i=0}^{t} G_{i j} g d_{i b}^{-\alpha}+N_{0}}\right)$

$T_{D U}\left(G_{i j}\right)=B \log _{2}\left(1+\frac{G_{i j} g d_{i i}^{-\alpha}}{G_{b} h d_{i b}^{-\alpha}+N_{0}}\right)$

$\mathrm{T}\left(\mathrm{G}_{\mathrm{ij}}\right)=\mathrm{T}_{\mathrm{DU}}\left(\mathrm{G}_{\mathrm{ij}}\right)$ when D2D user mode is active

$\mathrm{T}\left(\mathrm{G}_{\mathrm{ij}}\right)=\mathrm{T}_{\mathrm{CUE}}\left(\mathrm{G}_{\mathrm{ij}}\right)$ when Cellular mode is active

$\mathrm{T}\left(\mathrm{G}_{\mathrm{ij}}\right)$ denotes as data rate of the communication, and whereas $\mathrm{B}, \mathrm{N}_{0}, \mathrm{~d}_{\mathrm{ii}}, \mathrm{d}_{\mathrm{b}}, \mathrm{d}_{\mathrm{ib}}, \alpha, \mathrm{t}, \mathrm{h}$ and $\mathrm{g}$ represents bandwidth, white gaussian noise, distance between the two user of ith D2D pair, separation between the base station and cellular user, distance between the ith D2D transmitter to base station, path loss exponent, number of D2D pair, shadow fading coefficient, and statistical distributed random effect of fading in the D2D pair environment respectively.

Mode selection plays a vital role in the D2D communication. In order to achieve the mode selection, we consider the channel quality and the interference level with all fading or wireless issues. In the work, we adopt data rate metric for the mode selection of the communication.

When

$\mathrm{T}_{\mathrm{CUE}}>\mathrm{T}_{\mathrm{DU}}$; represents that Cellular user Mode is active $(\mathrm{m}=0)$

$\mathrm{T}_{\mathrm{DU}}>\mathrm{T}_{\mathrm{CUE}} ;$ represents tha $\mathrm{D} 2 \mathrm{D}$ user Mode is active $(\mathrm{m}=1)$

and

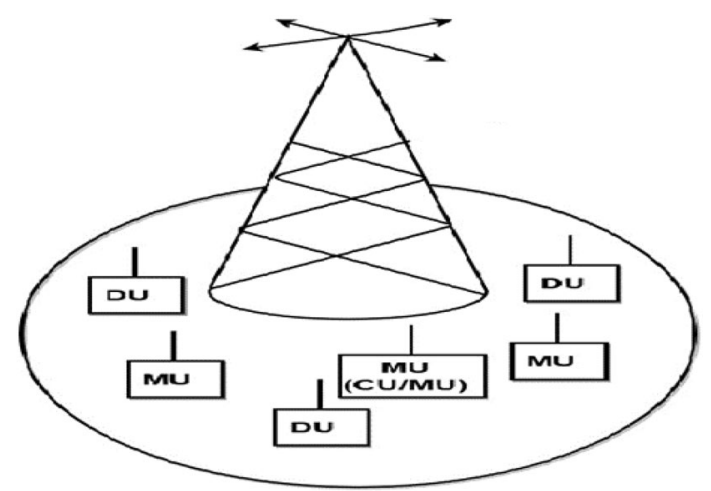

Fig. 1 Cellular architecture with different DU and MU 
$\mathrm{T}\left(\mathrm{G}_{\mathrm{ij}}\right)=\mathrm{mT}_{\mathrm{DU}}\left(\mathrm{G}_{\mathrm{ij}}\right)+(1-\mathrm{m}) \mathrm{T}_{\mathrm{CUE}}\left(\mathrm{G}_{\mathrm{ij}}\right)$

The total power during the communication is

$P=\sum_{n=1}^{t+A_{\text {cue }}} \sum_{i=1}^{t} \sum_{j=1}^{B} y_{i j n} G_{i j n} m_{i j n}+P_{c d}+P_{c c}$

whereas, $\sum \sum \sum y_{i j n} G_{i j n} m_{i j n}, \mathrm{P}_{c d}$ and $\mathrm{P}_{c c}$ denotes the total transmit power consumption, and the total circuit power consumption of DU and CUE respectively including battery backup

$\mathrm{P}_{\mathrm{cd}}=0 ;$ for $(\mathrm{m}=0)$

And $\mathrm{P}_{\mathrm{cc}}=0 ;$ for $(\mathrm{m}=1)$

In order to formulate the mathematical model of energy efficiency we use the Bit per joule metric and evaluate the optimal energy efficiency of the D2D communication after selecting the mode of the communication. The generalize formula of Energy efficiency is represented by $\eta_{\mathrm{EE}}$

$\eta_{E E}=\frac{\sum_{n=1}^{t+A_{\text {сие }}} \sum_{i=1}^{t} \sum_{j=1}^{B} y_{i j n} T\left(G_{i j n}\right) m_{i j n}}{\sum_{n=1}^{t+A_{\text {cue }}} \sum_{i=1}^{t} \sum_{j=1}^{B} y_{i j n} G_{i j n} m_{i j n}+P_{c d}+P_{c c}}$

Here, $T\left(G_{i j}\right)=\mathrm{mT}_{\mathrm{DU}}\left(\mathrm{G}_{\mathrm{ij}}\right)+(1-\mathrm{m}) \mathrm{T}_{\mathrm{CUE}}\left(\mathrm{G}_{\mathrm{ij}}\right)$ (for the D2D communication mode the value of $m$ should be one only), and

$\mathrm{y}_{\mathrm{ijn}} \in\{0,1\}$ shows that jth resource block is allocated to the ith of the MU with nth selected mode.

$\mathrm{m}_{\mathrm{ijn}} \in\{0,1\}$ shows $\mathrm{n}$ mode selection with $\mathrm{jth}$ resource block is allocated to the ith of the MU.

The D2D energy efficient optimization problem can mathematically represent as

$$
\underset{y_{i j n} G_{i j n} m_{i j n}}{\operatorname{Max}} \eta_{E E}
$$

Subject to. $\mathrm{m}_{\mathrm{ijn}} \in\{0,1\}, \forall_{\mathrm{i}} \in \mathrm{C}, \forall_{\mathrm{j}}=\mathrm{B}, \forall_{\mathrm{n}}=\mathrm{D}$

$\sum \mathrm{m}_{\mathrm{ijn}} \geq 1, \forall_{\mathrm{n}} \in \mathrm{C}$

$\sum \mathrm{m}_{\mathrm{ijn}}<1, \forall_{\mathrm{n}} \in \mathrm{D} \cap \mathrm{C}$

$\mathrm{y}_{\mathrm{ijn}} \in\{0,1\}, \forall_{\mathrm{i}} \in \mathrm{C}, \forall_{\mathrm{j}}=\mathrm{B}, \forall_{\mathrm{n}}=\mathrm{D}$

$\sum_{j=1}^{B} y_{i j n}=1, \forall_{i} \in C, \forall_{n} \in C$

$\sum_{i=1}^{t} \sum_{n=1}^{t+A_{\text {cue }}} y_{i j n} \leq 1, \forall_{j}=B$

$\mathrm{T}\left(\mathrm{G}_{\mathrm{ijn}}\right)=\mathrm{T}_{\mathrm{DU}}\left(\mathrm{G}_{\mathrm{ijn}}\right) \geq \beta, \forall \mathrm{y}_{\mathrm{ijn}} \in 1$

$\mathrm{G}_{\mathrm{b}} \mathrm{h} \mathrm{d}_{\mathrm{b}}^{-\alpha} \leq \bar{\lambda}, \forall \mathrm{y}_{\mathrm{ijn}} \in 1$

$\mathrm{G}^{\mathrm{min}}<\mathrm{G}_{\mathrm{ijn}} \leq \mathrm{G}^{\mathrm{max}}, \forall \mathrm{y}_{\mathrm{ijn}} \in 1$ whereas $\mathrm{G}^{\mathrm{min}}$ denotes the requirement of minimum transmit power of each DU for the purpose of the communication, $G^{\max }$ shows the maximum transmit power of each DU, and $\beta$ denotes the minimum rate requirement of each DU, $\lambda$ shows the threshold value of the allowed interference from $\mathrm{CU}$ when shared the same resources with corresponding DU.

\section{Proposed joint low power energy efficient resource management algorithm with mode selection}

Our proposed joint algorithm has two subsections. One is iterative algorithm for the energy efficiency named as Algorithm II and the second is iterative algorithm to attain the optimal low power with all constraints using the Lagrange dual function and multipliers named as Algorithm III. In the work, due to the self-realizing quality of the novel proposed formulated problem Eq. 11, the Algorithm I automatically recalled for getting the solution according to the selection of the D2D user and CUE.

In the Fig. 2, the logical flow process of the proposed Joint low power and energy efficient algorithm with resource management and mode selection is illustrated. The aim of the process is to provide a framework that reflects journey for achieving the low power and energy efficient system with the resource management and mode selection for the D2D communication. The process starts with the initialization of all the variables mentioned in the above Eqs. 1-21. After attaining all the variables for the D2D communication scenario, the Algorithms No. 2 comes into the picture. The analyzer can use the Algorithm No. 2 for the attaining the energy efficient resource management with mode selection including power control. Inhibit quality of the Algorithm no. 2 (Step number 4) enforce the use of Algorithm no. 1 for the mode selection purpose. If the $\mathrm{D} 2 \mathrm{D}$ mode is active, the process goes into the next step by following the Algorithm No. 3 to attain the optimized low power with low transmit power rate, data rate, and interference constraints with the use of lagrange dual function and lagrange multipliers otherwise go back into the first block for initiating the variables. The process would be complete after attaining the desired output from the proposed Joint Low power and energy efficient algorithm with resource management and mode selection.

\subsection{Mode selection}

In the mode selection, the selection of the D2D user and CUE is defined. In this approach it is decided by using the probabilistic theory that the particular Mobile user is going for the $\mathrm{D} 2 \mathrm{D}$ communication or not. 


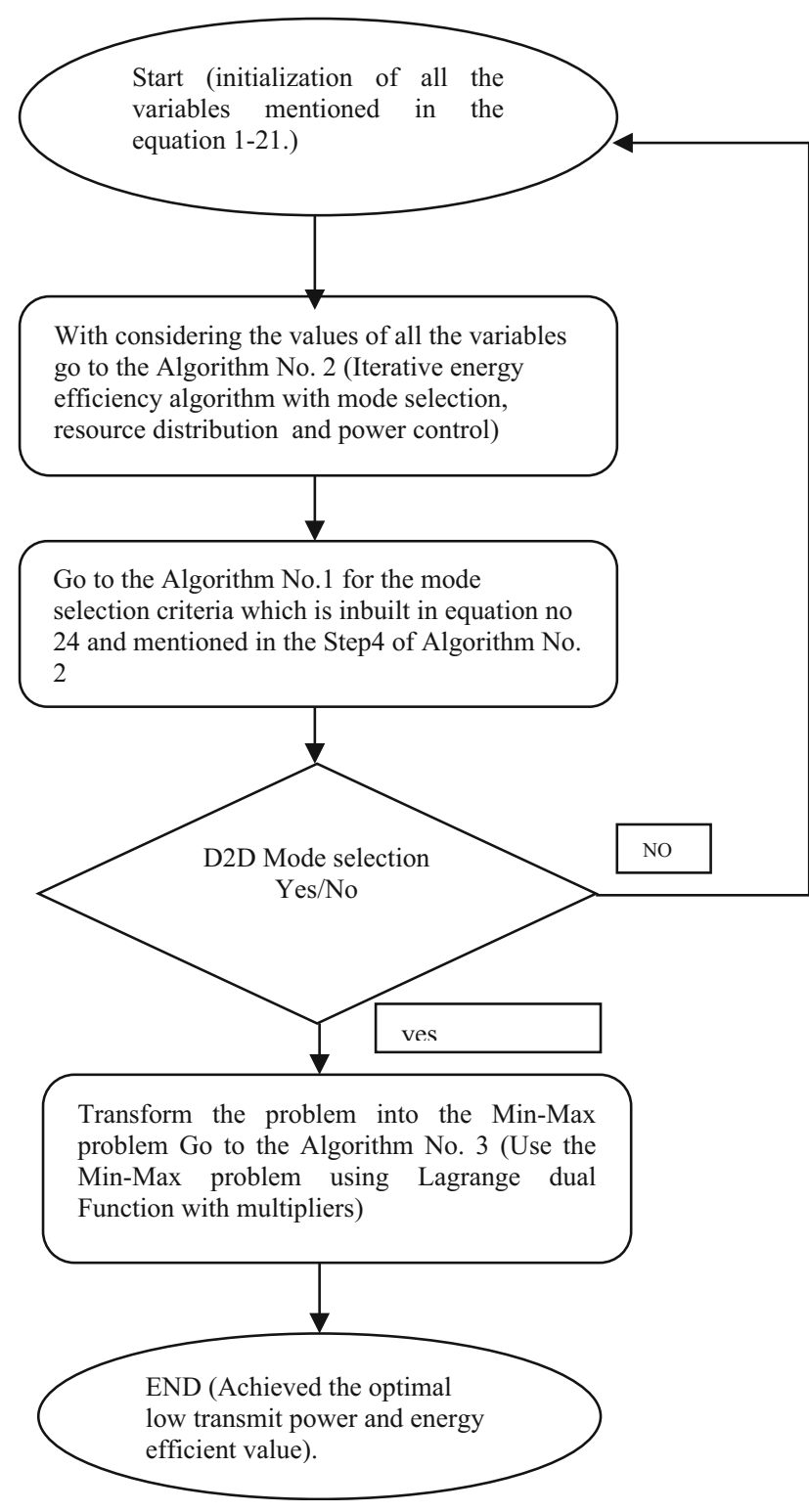

Fig. 2 Flow chart of proposed joint algorithm

\section{Algorithm I. Mode selection}

\begin{tabular}{|l|l|}
\hline \multicolumn{2}{|c|}{ Algorithm-I } \\
\hline Step 1 & $\begin{array}{l}\text { Calculate the total number of users that is } \\
\mathrm{t}+\mathrm{A}_{\text {cue. }}\end{array}$ \\
\hline Step 2 & Set $\mathrm{n}=1 ;$ \\
\hline Step 3 & $\begin{array}{l}\text { if }\left(\mathrm{T}_{\mathrm{DU}}>\mathrm{T}_{\mathrm{CUE}}\right) \\
\text { and }\left(\mathrm{T}_{\mathrm{DU}}>\beta\right), \text { then move to step } 5\end{array}$ \\
\hline Step 4 & else move to step 7 \\
\hline Step 5 & $\begin{array}{l}\text { if }\left(\mathrm{T}_{\mathrm{DU}}>\beta\right) \\
\text { set the value } \mathrm{m}_{\text {ijn }}=1 ;\end{array}$ \\
\hline Step 6 & else set the value $\mathrm{m}_{\text {ijn }}=0 ;$ \\
\hline Step 7 & $\begin{array}{l}\text { set } \mathrm{n}=\mathrm{n}+1 \text {; and } \text { Repeat step } 2 \text { to } 7 . \\
\text { until } \mathrm{n}=\mathrm{t}+\mathrm{A}_{\text {cue. }}\end{array}$ \\
\hline
\end{tabular}

Theorem 1 To prove

$\mathrm{T}\left(\mathrm{G}_{\mathrm{ijn}}\right)=\mathrm{T}_{\mathrm{DU}}\left(\mathrm{G}_{\mathrm{ijn}}\right)$

when D2D mode is selected or active.

Proof In this work the selection of mode depends upon the value of $\mathrm{T}_{\mathrm{DU}}$ and $\mathrm{T}_{\mathrm{CUE}}$

$\mathrm{T}_{\mathrm{CUE}}>\mathrm{T}_{\mathrm{DU}}$; represents that Cellular user Mode is active $(\mathrm{m}=0)$.

$\mathrm{T}_{\mathrm{DU}}>\mathrm{T}_{\mathrm{CUE}}$; represents that $\mathrm{D} 2 \mathrm{D}$ user Mode is active $(\mathrm{m}=1)$.

let assume that

$\mathrm{T}_{\mathrm{DU}}>\mathrm{T}_{\mathrm{CUE}}$ and $\mathrm{T}_{\mathrm{DU}}>\beta$

In this case the D2D user mode is active and value of $\mathrm{m}$ becomes one only.

From Eq. 7, we know that $\mathrm{T}\left(\mathrm{G}_{\mathrm{ijn}}\right)=\mathrm{mT}_{\mathrm{DU}}\left(\mathrm{G}_{\mathrm{ijn}}\right)+(1-\mathrm{m}) \mathrm{T}_{\mathrm{CUE}}\left(\mathrm{G}_{\mathrm{ijn}}\right)$.

By putting the value of $\mathrm{m}=1$

$$
\begin{aligned}
\mathrm{T}\left(\mathrm{G}_{\mathrm{ijn}}\right) & =1 \mathrm{~T}_{\mathrm{DU}}\left(\mathrm{G}_{\mathrm{ijn}}\right)+(1-1) \mathrm{T}_{\mathrm{CUE}}\left(\mathrm{G}_{\mathrm{ijn}}\right) \\
\mathrm{T}\left(\mathrm{G}_{\mathrm{ijn}}\right) & =1 \mathrm{~T}_{\mathrm{DU}}\left(\mathrm{G}_{\mathrm{ijn}}\right)+(0) \mathrm{T}_{\mathrm{CUE}}\left(\mathrm{G}_{\mathrm{ijn}}\right) \\
\mathrm{T}\left(\mathrm{G}_{\mathrm{ijn}}\right) & =\mathrm{T}_{\mathrm{DU}}\left(\mathrm{G}_{\mathrm{ijn}}\right)
\end{aligned}
$$

Hence proved.

\subsection{Iterative method for maximum energy efficiency}

Our main objective is to maximize the $\eta_{\mathrm{EE}}$ with subject to Eqs. 13-21

Let $\mathrm{k}$ represents the maximum value of $\eta_{\mathrm{EE}}, £$ represents the value of the constraints Eqs. 13-21. k may be defined as follows

$k=\underset{y_{i j n}, G_{i j n}, m_{i j n} \in \mathfrak{E}}{\operatorname{Max}}\left(\frac{\sum_{n=1}^{t+A c u e} \sum_{i=1}^{t} \sum_{j=1}^{B} y_{i j n} T\left(G_{i j n}\right) m_{i j n}}{\sum_{n=1}^{t+A c u e} \sum_{i=1}^{t} \sum_{j=1}^{B} y_{i j n} G_{i j n} m_{i j n}+P_{c d}+P_{c c}}\right)$.

Theorem 2 For the D2D communication the maximum $\mathrm{\eta}_{E E}$ with joint mode selection is achieved if and only if

$$
\begin{gathered}
\underset{y_{i j n}, G_{i j n}, m_{i j n} \in £}{\operatorname{Max}}\left\{\sum_{n=1}^{t+\text { Acue }} \sum_{i=1}^{t} \sum_{j=1}^{B} y_{i j n} T\left(G_{i j n}\right) m_{i j n}\right. \\
\left.-k\left(\sum_{n=1}^{t+A c u e} \sum_{i=1}^{t} \sum_{j=1}^{B} y_{i j n} G_{i j n} m_{i j n}+P_{c d}+P_{c c}\right)\right\}=0 .
\end{gathered}
$$

Proof In this case for the D2D communication, joint mode selection is done in Eq. 25. By considering the Eqs. 7, 10, and 25 we get

$$
\begin{aligned}
& \underset{y_{i j n}, G_{i j n}, m_{i j n} \in \mathfrak{£}}{\operatorname{Max}}\left\{\sum_{n=1}^{t+\text { Acue }} \sum_{i=1}^{t} \sum_{j=1}^{B} y_{i j n} T\left(G_{i j n}\right) m_{i j n}\right. \\
& \left.-k\left(\sum_{n=1}^{t+\text { Acue }} \sum_{i=1}^{t} \sum_{j=1}^{B} y_{i j n} G_{i j n} m_{i j n}+P_{c d}\right)\right\}=0 .
\end{aligned}
$$


Now, the maximum $\eta_{E E}$ is achieved if and if only Eq. 26 satisfies its conditions.

The proof of this statement is similar with the proof in Dinkelbach (1967). It can be observed from Eq. 26 and Dinkelbach (1967) that the fractional objective function represented in the subtractive form same as the case (Ye and Zhang 2015). To obtain the objective function which is in the fractional form is solve by analyzing its subtractive form. Similarly, we focus on the objective function in the subtractive form.

Let $\mathrm{k}_{\mathrm{r}}$ denotes the temporary $\eta_{\mathrm{EE}}$ and $\gamma_{1}$ denotes the convergence threshold value for the iterative algorithm.

Algorithm II: Iterative algorithm for resource allocation and power control with mode selection

\begin{tabular}{|c|c|}
\hline \multicolumn{2}{|r|}{ Algorithm-II } \\
\hline Step 1 & Set the number of iteration $\mathrm{r}=1$ and $\mathrm{k}_{\mathrm{r}}=0$; \\
\hline Step 2 & 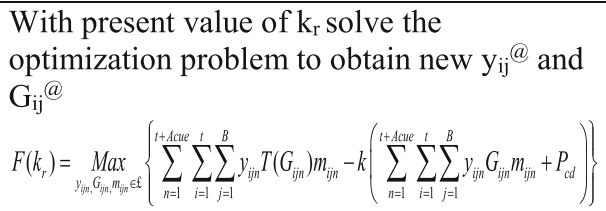 \\
\hline Step 3 & $k_{r}=\frac{\sum_{n=1}^{\text {Set }} \sum_{i=1}^{t+A c u e} \sum_{j=1}^{B} y_{i j n}^{@} T\left(G_{i j n}\right)^{@} m_{i j n}}{\sum_{n=1}^{t+A c u e} \sum_{i=1}^{t} \sum_{j=1}^{B} y_{i j n}^{@} G_{i j n}^{@} m_{i j n}+P_{c d}+P_{c c}}$ \\
\hline Step 4 & $\begin{array}{l}\text { Go to the Algorithm I for getting the value } \\
\text { of } \mathrm{m}_{\mathrm{ijn}} \text { and after attaining the value } \mathrm{T}\left(\mathrm{G}_{\mathrm{ijn}}\right)= \\
\mathrm{T}_{\mathrm{DU}}\left(\mathrm{G}_{\mathrm{ijn}}\right) \text { go to the Step } 5 \text {. }\end{array}$ \\
\hline Step 5 & $\begin{array}{l}\text { If }\left(\left|\mathrm{F}\left(\mathrm{k}_{\mathrm{r}}\right)\right| \geq \gamma_{1}\right) \\
\{\text { Set } \mathrm{r}=\mathrm{r}+1 ; \\
\text { Repeat step } 2,3,4 \text { and } 5\}\end{array}$ \\
\hline Step 6 & else $\{$ end $\}$. \\
\hline
\end{tabular}

\subsection{Lagrange dual method use for optimum low power and energy efficient, resource allocation algorithm with mode selection}

In this section we are using the Lagrange dual method for attaining the optimum minimum power for the above mentioned problem after following the (Algorithm I and II) mode selection, resource and power allocation. By using the Eq. 24, with considering Eqs. 19-21, we can formulate the optimum power in Lagrangian form and can be written as follows:whereas $\mathrm{u}, \mathrm{v}, \mathrm{w}$ are the multipliers of transmission power constraint, data rate and allowed interference respectively. The Low Power optimization can be obtained by using Lagrange dual problem shown as

$\mathrm{L}\left(\mathrm{G}_{\mathrm{ij}}, \mathrm{u}, \mathrm{v}, \mathrm{w}\right)=\mathrm{T}_{\mathrm{DU}}\left(\mathrm{G}_{\mathrm{ij}}\right)$

$-\mathrm{k}\left(\mathrm{G}_{\mathrm{ij}}+\mathrm{P}_{\mathrm{cd}}\right)-\mathrm{u}\left(\mathrm{G}_{\mathrm{ij}}-\mathrm{G}^{\mathrm{max}}\right)$

$-\mathrm{v}\left(\beta-\mathrm{T}_{\mathrm{DU}}\left(\mathrm{G}_{\mathrm{ij}}\right)\right)-\mathrm{w}\left(\bar{\lambda}-\bar{\lambda}_{\mathrm{ij}}\right)$

$\min _{u, v, w} \max _{G_{i j}} L\left(G_{i j}, u, v, w\right) ;$ s.t.u, $, v, w \geq 0$

By using the present Lagrange multipliers $\mathrm{u}, \mathrm{v}$, w, and Eqs. 1 and 22, we can find an optimal power allocation method shown as

$G_{i j}=\left[\frac{(1+v) B}{(k+u) \ln 2}-\frac{(1+w)\left(G_{b} h d_{b}^{-\alpha}+N_{0}\right)}{g d_{i i}^{-\alpha}}\right]^{+}$

where $[\mathrm{x}]^{+}=\max [0, \mathrm{x}]$, for updating the lagrange multipliers we use gradient method

$$
\begin{aligned}
\mathrm{u}(\mathrm{r}+1) & =\mathrm{u}(\mathrm{r})+\Omega_{1}\left(\mathrm{G}_{\mathrm{max}}-\mathrm{G}_{\mathrm{ij}}\right) \\
\mathrm{v}(\mathrm{r}+1) & =\mathrm{v}(\mathrm{r})+\Omega_{2}\left(\mathrm{~T}_{\mathrm{DU}}\left(\mathrm{G}_{\mathrm{ij}}\right)-\beta\right) \\
\mathrm{w}(\mathrm{r}+1) & =\mathrm{w}(\mathrm{r})+\Omega_{3}\left(\bar{\lambda}_{\mathrm{ij}}-\bar{\lambda}\right)
\end{aligned}
$$

whereas $\Omega_{1}, \Omega_{2}, \Omega_{3}$ are the step sizes.

The proposed algorithm for the optimal low power allocation using Lagrange dual function is mentioned in the Algorithm III.

Let $r_{\max }$ is the maximum number of iterations and $\gamma_{2}$ denotes the maximum convergence threshold value for the iterative algorithm. 


\begin{tabular}{|c|c|}
\hline \multicolumn{2}{|c|}{ Algorithm III } \\
\hline \multicolumn{2}{|r|}{ Algorithm-III } \\
\hline $\begin{array}{l}\text { Step } \\
1\end{array}$ & $\begin{array}{l}\text { After following the Algorithm-I and } \\
\text { Algorithm-II go to the step2 }\end{array}$ \\
\hline \multirow{3}{*}{$\begin{array}{l}\text { Step } \\
2\end{array}$} & Set the initial value of $\mathrm{u}, \mathrm{v}, \mathrm{w}$ for $\mathrm{r}=0$; \\
\hline & 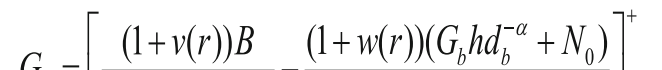 \\
\hline & $G_{i j}=\left[\begin{array}{ll}(k+u(r)) \ln 2 & g d_{i i}^{-\alpha}\end{array}\right.$ \\
\hline $\begin{array}{l}\text { Step } \\
3\end{array}$ & Find the value of $\mathrm{G}_{\mathrm{ij}}$ by using equation number \\
\hline $\begin{array}{l}\text { Step } \\
4\end{array}$ & $\begin{array}{l}\text { Update the Lagrangian multipliers } \\
\mathrm{u}(\mathrm{r}+1)=\left[\mathrm{u}(\mathrm{r})-\Omega_{1}\left(\mathrm{G}_{\mathrm{ij}}-\mathrm{G}^{\max }\right)\right]^{+} \\
\mathrm{v}(\mathrm{r}+1)=\left[\mathrm{v}(\mathrm{r})-\Omega_{2}\left(\beta-\mathrm{T}_{\mathrm{DU}}\left(\mathrm{G}_{\mathrm{ij}}\right)\right)\right]^{+} \\
\mathrm{w}(\mathrm{r}+1)=\left[\mathrm{w}(\mathrm{r})-\Omega_{3}\left(\lambda-\lambda_{\mathrm{ij}}\right)\right]^{+}\end{array}$ \\
\hline $\begin{array}{l}\text { Step } \\
5\end{array}$ & $\begin{array}{l}\text { if } \\
|u(r+1)-u(r)|+|v(r+1)-v(r)|+|w(r+1)-w(r)| \leq \gamma_{2} \\
\left.\text { return } \mathrm{G}_{\mathrm{ij}}{ }^{*}=\mathrm{G}_{\mathrm{ij}}\right\} \\
\text { else } \\
\text { set } \mathrm{r}=\mathrm{r}+1 ;\end{array}$ \\
\hline $\begin{array}{l}\text { Step } \\
6\end{array}$ & $\begin{array}{l}\text { Repeat the step } 2,3,4,5 \text { until } \\
|u(r+1)-u(r)|+|v(r+1)-v(r)|+|w(r+1)-w(r)|>\gamma_{2} \\
\text { or } \mathrm{r}=\mathrm{r}_{\max } \text {. }\end{array}$ \\
\hline
\end{tabular}

By following the above mentioned proposed algorithms one can achieve the low power energy efficient resource allocation with mode selection.

\section{Results and discussions}

In this section we evaluate the performance analysis of our proposed algorithm for $\mathrm{D} 2 \mathrm{D}$ communication. We consider the single cell having $\mathrm{A}_{\text {cue }}, \mathrm{t}$ number of CUE and DU respectively within 200-m radius and the average power value of all D2D users are same. For the purpose of analysis, we considered the system parameters which are mentioned in Table 1.

By considering the above mentioned system parameters, first of the all, the mode selection is achieved which ensures that the particular mobile user is working as a D2D user or not by using Algorithm 1 and by following the Algorithms 2 and 3, we get the performance evaluation of low power energy efficient resource allocation with mode selection. Figures 2, 3, and 4 are representations are the outcomes of the Algorithm 2, and Fig. 5 illustrates the final step of proposed work using the algorithm 3 .

In Fig. 3, the EE versus number of iteration with $t$ different number of D2D users (DU) is illustrated. It is observed that the Energy efficiency of the proposed algorithm increases with the number DU. As the number of
D2D user increases we achieved the higher value of EE (Jiang et al. 2016). It is also observed that only 2 iteration process requires gaining the maximum energy efficiency (EE) value. In our proposed algorithm no. 2, by adding the optimal power with low transmission rate, data rate, and interference constraints using the (min-max problem) Lagrange dual function with Lagrange multiplier (Mensah et al. 2016), we achieve better performance that is shown in the Fig. 6.

In Fig. 4, the energy efficiency versus distance between D2D users with different value of power value is represented. It is observed in the plot that the lower value of transmit power have the high energy efficiency and the energy efficiency is decreases as distance between the D2D user increases.

In Fig. 5, the data rate versus distance between D2D users with different value of power value is shown. Figure 5 shows the mutual relationship between the D2D distance and data rate. Higher power has high data rate and as the distance increases the data rate will decrease. As per Fig. 4 the low power object achieves the high energy efficiency and in the Fig. 5, it is shown that the high power attains the higher date rate, due to these behavioral representation of the Algorithm 2 requires the need of the importance to choose the optimal power value with including the data rate and all other constraints, which is further carried out by the Algorithm no 3. and Fig. 6 conclude the final representation of our proposed algorithm.

In Fig. 6, (EE) versus maximum transmitting power of D2D user is represented. Based on our proposed algorithm the resource allocation with mode selection, low power allocation strategies, and energy efficiency of the DU can be examined and is drawn in the figure. It is observed that higher value of maximum power threshold is required to achieve the greater energy efficiency to attain the optimal low power value $G^{\max }$. After numerical analysis the threshold value $0.002 \mathrm{w}$ is obtained and set as a threshold for the proposed algorithm. It is observed that the energy

Table 1 System parameter

\begin{tabular}{ll}
\hline Cell radius & $200 \mathrm{~m}$ \\
D2D distance & $20 \mathrm{~m}$ \\
Noise & $-174 \mathrm{dbm} / \mathrm{Hz}$ \\
Path loss exponent & 4 \\
Shadow fading/random fading effect & $8 \mathrm{db}$ \\
$\mathrm{G}^{\text {max }}$ & $0.5 \mathrm{~W}$ \\
Threshold & $50 \mathrm{~mW}$ \\
Total circuit power consumption $\left(\mathrm{P}_{\mathrm{cd}}\right)$ & $0.1 \mathrm{~W}$ \\
Base station transmission power $\left(\mathrm{G}_{\mathrm{b}}\right)$ & $1 \mathrm{~W}$ \\
Bandwidth (B) & $1 \mathrm{MHz}$ \\
\hline
\end{tabular}




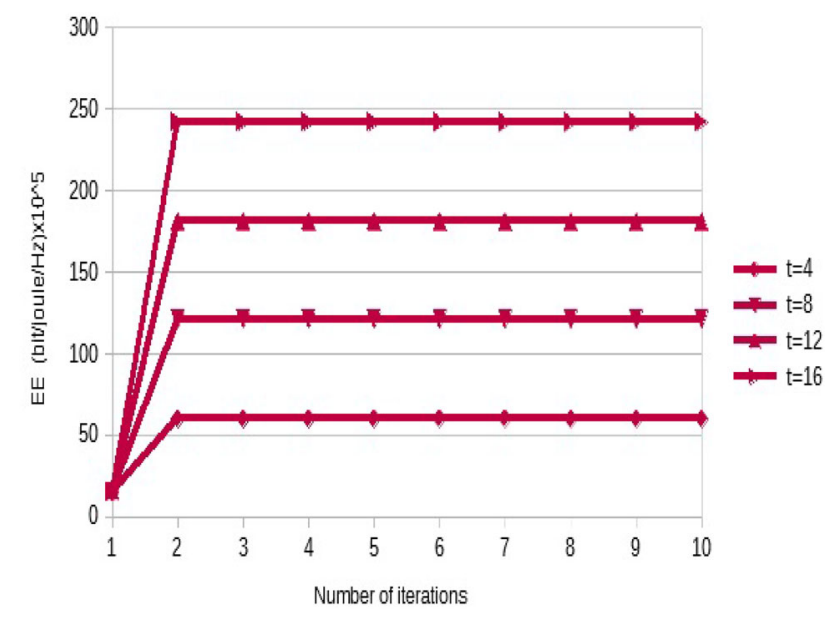

Fig. 3 EE versus iterations number

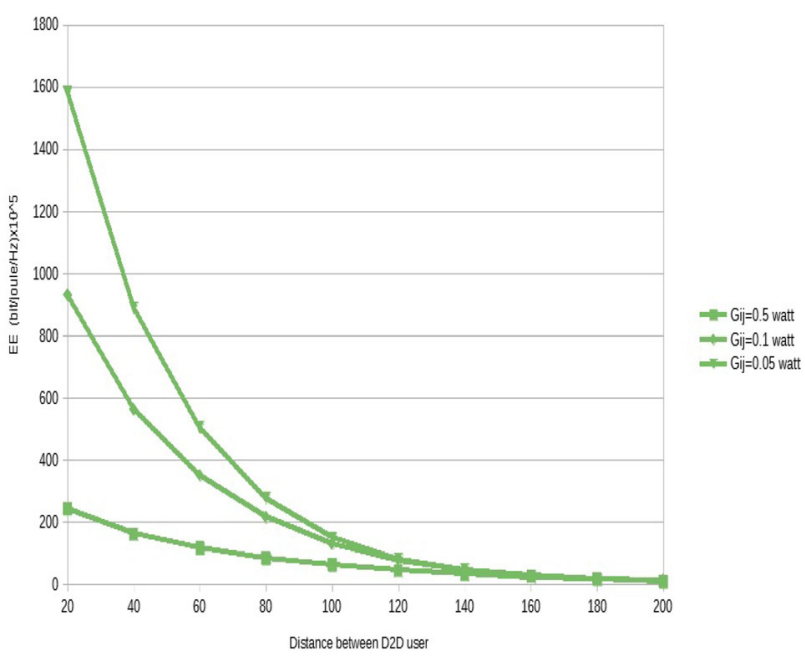

Fig. 4 EE versus distance between D2D users

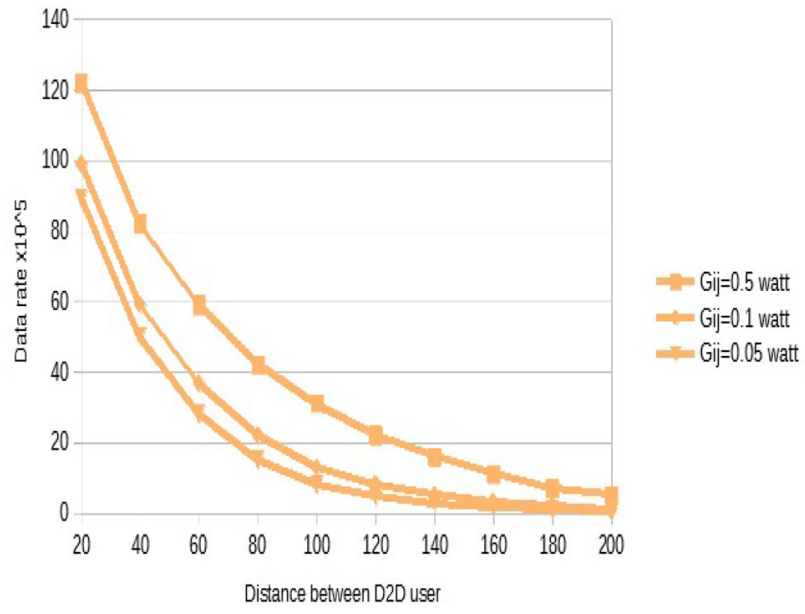

Fig. 5 Data rate versus distance between D2D users

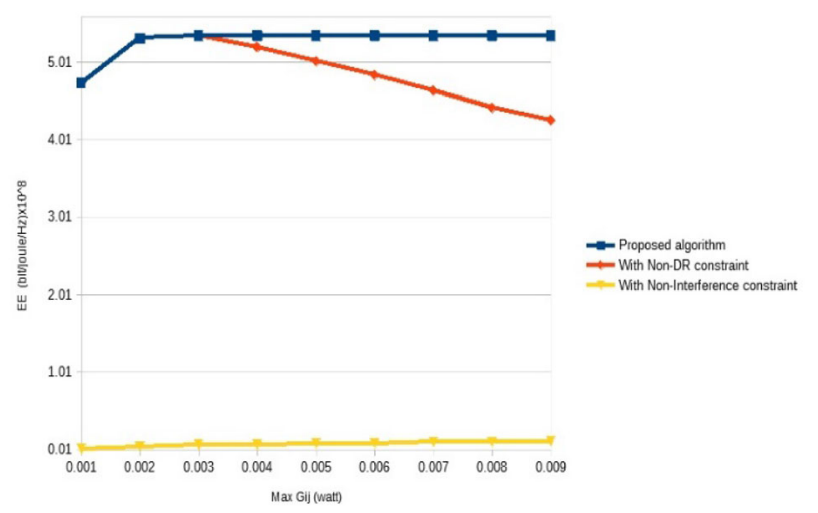

Fig. 6 Illustrate that our proposed algorithm, provide better performance in the terms of low transmit power value and energy efficiency

efficiency increases with the increase of $G^{\max }$. When the proposed algorithm reaches the threshold value, the energy efficiency provides a fixed value which doesn't varies with further raising the value of $G^{\max }$ and we can get optimal low power energy efficient value.

In the Fig. 6, we can compare the performance evaluation of mentioned three scenarios proposed algorithm, Non-DR constraint, and Non- Interference constraint respectively. It is clearly visualized that proposed algorithm provides better energy efficiency (max value $5.32 \times 10^{8} \mathrm{bit} / \mathrm{J} / \mathrm{Hz}$ ) with increase in the maximum transmit power value comparison to the Non-DR constraint, and Non- Interference constraint. It is analyzed that the addition of optimal power with low transmit rate, data rate, and interference constraints improves our algorithm performance in comparison to the Non-DR constraint, and NonInterference constraint.

It is seen from the Table 2 and numerical results that our proposed algorithm achieve better results from the prevailed researches carrying resource allocation with power management.

\section{Conclusion}

In this paper, we proposed the novel low power energy efficient algorithm resource allocation algorithm with mode selection by formulating the optimal joint problem of mode selection, resource allocation, and power control using equivalent subtraction form of a fractional function problem to maximize the energy efficiency and further use the Lagrangian dual function with Lagrangian multipliers for attaining the optimal transmit power with low transmit power rate, data rate, and interference constraints. In the work, we conduct the numerical analysis for the validation of the algorithm, and the numerical result shows that with low power of $0.002 \mathrm{~W}$ we achieve the maximum energy 
Table 2 Comparative table

\begin{tabular}{|c|c|c|c|}
\hline Algorithm & References & Characteristic parameters & Improvement \\
\hline $\begin{array}{r}\text { Distributed } \\
\text { algorithm }\end{array}$ & Liu (2016) & Optimal mode selection & Average throughput increases with transmit power \\
\hline Bandit model & $\begin{array}{l}\text { Maghsudi and } \\
\text { Niyato } \\
(2017)\end{array}$ & Mode selection & Improves transmission mode selection \\
\hline $\begin{array}{l}\text { Heuristic } \\
\text { algorithm }\end{array}$ & $\begin{array}{l}\text { Jiang et al. } \\
\text { (2018) }\end{array}$ & $\begin{array}{l}\text { Resource allocation and } \\
\text { power control }\end{array}$ & Dynamic power control with resource allocation \\
\hline $\begin{array}{l}\text { SE algorithm } \\
\text { with pilot } \\
\text { allocation }\end{array}$ & $\begin{array}{l}\text { Ghazanfari } \\
\text { et al. (2018) }\end{array}$ & Power control & Optimize the power control with capacity \\
\hline $\begin{array}{l}\text { Iterative } \\
\text { algorithm }\end{array}$ & $\begin{array}{l}\text { Jiang et al. } \\
\quad(2016)\end{array}$ & $\begin{array}{l}\text { Resource allocation and } \\
\text { power control }\end{array}$ & Energy Efficiency with resource allocation and power control \\
\hline \multirow[t]{3}{*}{$\begin{array}{l}\text { Proposed } \\
\text { algorithm }\end{array}$} & & $\begin{array}{l}\text { Mode selection, resource } \\
\text { allocation, and power } \\
\text { control }\end{array}$ & $\begin{array}{l}\text { Energy efficiency with mode selection, resource allocation and power } \\
\text { control }\end{array}$ \\
\hline & & & $\begin{array}{l}\text { Optimize the power with Interference, data rate, low transmit power } \\
\text { constraints, using Lagrange method }\end{array}$ \\
\hline & & & $\begin{array}{l}\text { In the proposed work, the mode selection, resource allocation and power } \\
\text { control done by using one mathematical function to obtain the Energy } \\
\text { efficiency. Interference data rate, low transmit power constraints in the } \\
\text { optimization provides an improvement in the results from the past } \\
\text { researches (Liu 2016; Maghsudi and Niyato 2017; Jiang et al. } \\
\text { 2016, 2018; Ghazanfari et al. 2018) }\end{array}$ \\
\hline
\end{tabular}

efficiency of $5.32 \times 10^{8} \mathrm{bit} / \mathrm{J} / \mathrm{Hz}$ along with the mode selection and resource allocation, and we get the improvement in the energy efficient joint resource allocation and power control for D2D communication (Jiang et al. 2016) and related research domain of the optimal energy efficient resource management for the D2D communication with advancement in the terms of optimal low power with maintaining the effectiveness of the communication considering the Interference, data rate, low transmit power constraints, and achieve the low power energy efficient resource allocation algorithm with mode selection for the D2D communication underlay in-band cellular networks. Our novel proposed algorithm can be further used in the low power and energy efficient devices for the D2D communication as an Integral element of IoT applications and $5 \mathrm{G}$.

\section{References}

Awan AY, Ali M, Naeem M, Qamar F, Sial MN (2020) Joint network admission control, mode assignment, and power allocation in energy harvesting aided D2D communication. IEEE Trans Ind Inf 16(3):1914-1923. https://doi.org/10.1109/TII.2019.2922667

Azam M (2016) Joint admission control, mode selection, and power allocation in D2D communication systems. IEEE Trans Veh Technol 65(9):7322-7333. https://doi.org/10.1109/TVT.2015. 2487220
Cai Y, Ni Y, Zhang J, Zhao S, Zhu H (2019) Energy efficiency and spectrum efficiency in underlay device-to-device communications enabled cellular networks. China Commun 16(4):16-34

Chettri L, Bera R (2020) A comprehensive survey on internet of things (IoT) toward 5G wireless systems. IEEE IoT J 7(1):16-32

Dinkelbach W (1967) On nonlinear fractional programming. Manag Sci 13(7):492-498

Fan L, Dong Z, Yuan P (2017) The capacity of device-to-device communication underlaying cellular networks with relay links. IEEE Access 5:16840-16846

Feng D, Jiang C, Lim G, Cimini LJ, Feng G, Li G (2013) A survey of energy-efficient wireless communications. IEEE Commun Surv Tutor 15:167-178

Fodor G, Roger S, Rajatheva N, Slimane SB, Svensson T, Popovski P, Silva JMBD, Ali S (2016) An overview of device-to-device communications technology components in METIS. IEEE Access 4:3288-3299

Gandotra P, Jha RK, Jain S (2017) A survey on device-to-device (D2D) communication: architecture and security issues. J Netw Comput Appl 78:9-29

Gao C, Sheng X, Tang J, Zhang W, Zou S, Guizani M (2014) Joint mode selection, channel allocation and power assignment for green device-to-device communications. In: 2014 IEEE international conference on communications (ICC), Sydney, NSW, pp 178-183. https://doi.org/10.1109/ICC.2014.6883315.

Ghazanfari A, Björnson E, Larsson EG (2018) Power control for D2D underlay in multi-cell massive MIMO networks. In: WSA 2018; 22nd international ITG workshop on smart antennas, Bochum, Germany, pp 1-6

Han C, Harrold T, Armour S, Krikidis I, Videv S, Grant PM, Haas H, Thompson J, Ku I, Wang C-X, Le TA, Nakhai M, Zhang J, Hanzo L (2011) Green radio: radio techniques to enable energyefficient wireless networks. IEEE Commun Mag 49:46-54

Han L, Zhang Y, Zhang X, Mu J (2019) Power control for full-duplex D2D communications underlaying cellular networks. IEEE 
Access 7:111858-111865. https://doi.org/10.1109/ACCESS. 2019.2934479

Hoyhtya M, Apilo O, Lasanen M (2018) Review of latest advances in 3GPP standardization: D2D communication in 5G systems and its energy consumption models. Future Internet 10:3

Huang W et al (2019) Joint power, altitude, location and bandwidth optimization for UAV with underlaid D2D communications. IEEE Wirel Commun Lett 8(2):524-527. https://doi.org/10. 1109/LWC.2018.2878706

Jiang Y, Liu Q, Zheng F, Gao X, You X (2016) Energy-efficient joint resource allocation and power control for D2D communications. IEEE Trans Veh Technol 65(8):6119-6127. https://doi.org/10. 1109/TVT.2015.2472995

Jiang F, Wang B, Sun C et al (2018) Resource allocation and dynamic power control for D2D communication underlaying uplink multi-cell networks. Wirel Netw 24:549-563. https://doi.org/ 10.1007/s11276-016-1351-7

Lee J, Lee JH (2019) Performance analysis and resource allocation for cooperative D2D communication in cellular networks with multiple D2D pairs. IEEE Commun Lett 23(5):909-912. https:// doi.org/10.1109/LCOMM.2019.2907252

Liu Y (2016) Optimal mode selection in D2D-enabled multibase station systems. IEEE Commun Lett 20(3):470-473. https://doi. org/10.1109/LCOMM.2015.2514100

Maghsudi S, Niyato D (2017) On transmission mode selection in D2D-enhanced small cell networks. IEEE Wirel Commun Lett 6(5):618-621. https://doi.org/10.1109/LWC.2017.2723558

Mensah KK, Chai R, Bilibashi D, Gao F (2016) Energy efficiency based joint cell selection and power allocation scheme for HetNets. Digit Commun Netw 2(4):184-190. https://doi.org/10. 1016/j.dcan.2016.11.004
Shalmashi S, Björnson E, Ben Slimane S, Debbah M (2014, Closedform optimality characterization of network-assisted device-todevice communications. In: 2014 IEEE wireless communications and networking conference (WCNC), Istanbul, pp 508-513. https://doi.org/10.1109/WCNC.2014.6952080

Sheng M, Li Y, Wang X, Li J, Shi Y (2016) Energy efficiency and delay tradeoff in device-to-device communications underlaying cellular networks. IEEE J Sel Areas Commun 34:92-106

Wang F, Xu C, Song L, Han Z (2014) Energy-efficient resource allocation for device-to-device underlay communication. IEEE Trans Wirel Commun 14(4):2082-2092. https://doi.org/10.1109/ TWC.2014.2379653

Wang M, Gao H, Su X, Tiejun LV (2016) Joint channel allocation, mode selection and power control in D2D-enabled femtocells. In: MILCOM 2016-2016 IEEE Military Communications Conference, Baltimore, MD, pp. 454-459. https://doi.org/10. 1109/MILCOM.2016.7795369

Wang H, Chen J, Ding G, Wang S (2018) D2D Communications underlaying UAV-assisted access networks. IEEE Access 6:46244-46255. https://doi.org/10.1109/ACCESS.2018.2865629

Xu L, Jiang C, Shen Y, Quek TQS, Han Z, Ren Y (2016) Energy efficient D2D communications: a perspective of mechanism design. IEEE Trans Wirel Commun 15:7272-7285

Ye J, Zhang YJ (2015) A guard zone based scalable mode selection scheme in D2D underlaid cellular networks. In: 2015 IEEE international conference on communications (ICC), London, pp 2110-2116. https://doi.org/10.1109/ICC.2015.7248637

Publisher's Note Springer Nature remains neutral with regard to jurisdictional claims in published maps and institutional affiliations. 